\title{
Vortices in self-bound dipolar droplets
}

\author{
André Cidrim, ${ }^{1,2}$ Francisco E. A. dos Santos, ${ }^{1}$ Emanuel A. L. Henn, ${ }^{2}$ and Tommaso Macrì ${ }^{3}$ \\ ${ }^{1}$ Departamento de Física, Universidade Federal de São Carlos, 13565-905 São Carlos, São Paulo, Brazil \\ ${ }^{2}$ Instituto de Física de São Carlos, Universidade de São Paulo, C.P. 369, 13560-970 São Carlos, SP, Brazil \\ ${ }^{3}$ Departamento de Física Teórica e Experimental, Universidade Federal do Rio Grande do Norte, \\ and International Institute of Physics, Natal-RN, Brazil
}

\begin{abstract}
Quantized vortices have been observed in a variety of superfluid systems, from ${ }^{4} \mathrm{He}$ to condensates of alkali-metal bosons and ultracold Fermi gases along the BEC-BCS crossover. In this article we study the stability of singly quantized vortex lines in dilute dipolar self-bound droplets. We first discuss the energetic stability region of dipolar vortex excitations within a variational ansatz in the generalized nonlocal Gross-Pitaevskii functional that includes quantum fluctuation corrections. We find a wide region where stationary solutions corresponding to axially-symmetric vortex states exist. However, these singly-charged vortex states are shown to be unstable, either by splitting the droplet in two fragments or by vortex-line instabilities developed from Kelvin-wave excitations. These observations are the results of large-scale fully three-dimensional simulations in real time. We conclude with some experimental considerations for the observation of such states and suggest possible extensions of this work.
\end{abstract}

PACS numbers: 67.10.Ba, 67.10.Fj, 67.85.Bc, 67.85.De, 67.85.Fg, 67.85.Hj

\section{INTRODUCTION}

Quantized vortices are a direct manifestation of the genuine quantum behavior of superfluid systems. A prime example is superfluid helium, which has been widely studied over the past decades. Ultracold quantum gases offer the possibility to investigate vortex properties in a complementary regime in terms of particle numbers, interaction strength, and range [1, 2] with either bosonic [3] or fermionic [4] atoms. Dipolar condensates may also display vortex excitations with peculiar properties as a consequence of the long range and anisotropy of the interactions [5] 8 . For a three-dimensional condensate with a vortex line and in the presence of a periodic potential, the spectrum of transverse modes may display a rotonlike minimum [9, 10, which destabilizes the straight vortex and leads to a transition from vortex into helical or snakelike configurations [11, 12].

Theoretical models of superfluid states of ultracold gases at zero temperature are usually based on wellestablished mean-field approximations which accurately describe experiments [13, ranging from analytic treatments and variational approaches to full numerical simulations. Corrections beyond the mean-field picture have been measured for strongly interacting Bose gases [14] and for ultracold fermions along the BCS-BEC crossover [15] and compared with ab initio quantum Monte Carlo calculations.

The recent observation of ultradilute self-bound droplets both in dipolar condensates [16- 22 , as well as in two-component Bose mixtures 23, together with a combined theoretical effort, established the importance of the fundamental role of quantum fluctuations in ultracold atomic systems 24 38. Yet, no work has investigated the presence and stability of vortex states in self-bound droplets in ultradilute liquids.

Helium droplets hosting several quantized vortices have been recently observed 39 and studied in detail theoretically [40 43, both in pure samples and in the presence of impurities. The scales are, nevertheless, completely different. Helium droplets can be easily taken as a homogeneous, infinite superfluid background since vortices are much smaller compared to the system size (given the large interaction strengths).

Droplets in quantum ferrofluids are very anisotropic, and vortex cores are expected to have a size comparable to that of the whole droplet. Here we address the issue of stability and dynamics of singly quantized vortex lines in dipolar droplets for a wide range of dipolar interaction strengths and particle numbers. We carry out large-scale fully three-dimensional simulations, which allow for an efficient determination of energies and shapes of droplets as well as their dynamics. We find a strong anisotropy of such droplets, very elongated along the polarization axis. For small particle numbers, droplets are dynamically unstable towards splitting in two droplets where angular momentum is redistributed into surface collective excitations 44]. For larger sizes we do not observe splitting, yet vortex lines display bending for long times [12, 45 48]. We conclude with a discussion of a possible experimental implementation and observation of our findings with current experimental setups.

\section{METHODS}

The dynamics of an untrapped dipolar Bose-Einstein condensate is described by a generalized non-local GrossPitaevskii equation,

$$
i \hbar \dot{\psi}=\left(-\frac{\hbar^{2} \nabla^{2}}{2 m}+\mathcal{K}_{\text {int }}(\mathbf{r})+g_{\mathrm{LHY}}|\psi|^{3}\right) \psi,
$$

where $\psi(\mathbf{r}, t)$ is the BEC wavefunction. $\mathcal{K}_{\text {int }}(\mathbf{r})=$ $g_{c}|\psi(\mathbf{r})|^{2}+\int \mathrm{d} \mathbf{r}^{\prime} V_{\mathrm{dd}}\left(\mathbf{r}-\mathbf{r}^{\prime}\right)\left|\psi\left(\mathbf{r}^{\prime}\right)\right|^{2}$ describes the con- 

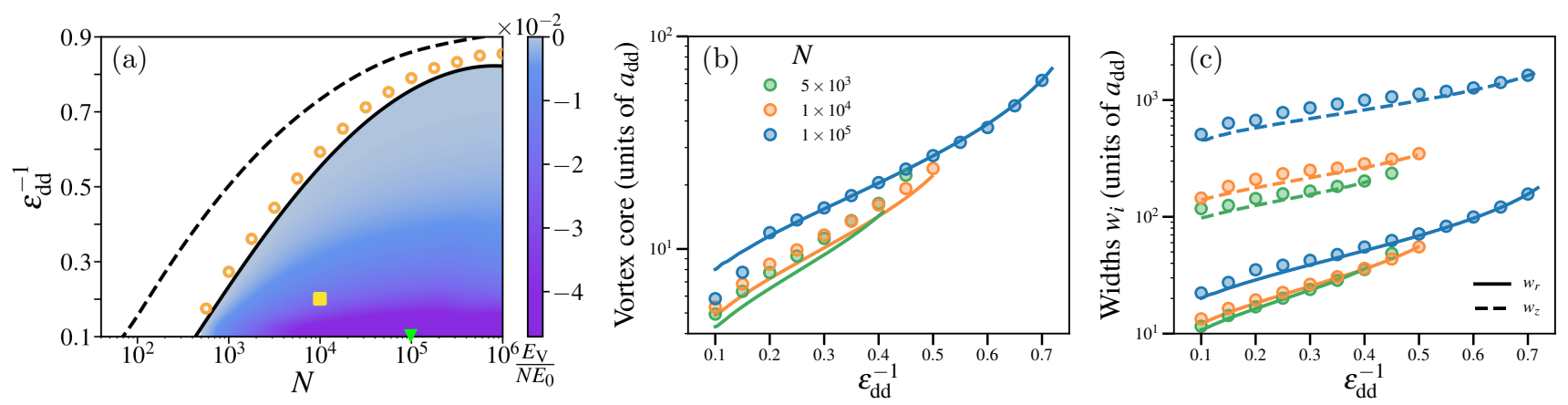

FIG. 1. (a) Stability diagram of an axisymmetric droplet with a vortex line. Density plot of the energy per particle of selfbound solutions hosting a vortex as a function of $\varepsilon_{\mathrm{dd}}^{-1}$ and $N$ calculated using the variational Gaussian wave function 22. The thick black line corresponds to $E_{\mathrm{V}}=0$. Energy is in units of $E_{0}=\hbar^{2} /\left(m a_{\mathrm{dd}}^{2}\right)$. The yellow square and the green triangle correspond to $\left(N, \varepsilon_{\mathrm{dd}}^{-1}\right)=\left(10^{4}, 0.2\right)$ and $\left(10^{5}, 0.1\right)$ coordinates, respectively, used in Fig. 2 to characterize the dynamics. (b), (c) Characterization of droplets hosting a vortex line. (b) Size of the vortex core scaled by the dipolar length as a function of $\varepsilon_{\mathrm{dd}}^{-1}$ and for $N=5 \times 10^{3}, 10^{4}, 10^{5}$ particle numbers. Full dots correspond to energy minima of the Gross-Pitaevskii energy functional. (c) Longitudinal $\left(w_{z}\right)$ and horizontal $\left(w_{r}\right)$ droplet size as a function of $\varepsilon_{\mathrm{dd}}^{-1}$ for the same particle numbers as in (b). In (b) the vortex core size is defined as the distance from the origin to the point where the density reaches $90 \%$ of its maximum. In (c) the radial (axial) width is defined as the mean length $w_{r(z)} \equiv\left(w_{i}+w_{e}\right) / 2$, where $w_{i}$ and $w_{e}$ are the distances between the points where the density reaches, respectively, $90 \%$ and $10 \%$ of its maximum value along the radial direction (along the vertical, peak-density line). In all panels lines are variational results, and dots are numerical results for stationary solutions of Eq. (1), imposing a vortex with azimuthal symmetry (see text).

tact and dipolar mean-field interaction of the condensate. Here $g_{c}=4 \pi a_{s} \hbar^{2} / m$ is the contact interaction strength, with $a_{s}$ being the $s$-wave scattering length, and $V_{\mathrm{dd}}(\mathbf{r})=\frac{C_{\mathrm{dd}}}{4 \pi} \frac{1-3 \cos ^{2} \theta}{r^{3}}$ is the dipolar potential. $C_{\mathrm{dd}}=$ $\mu_{0} \mu^{2} \equiv \frac{12 \pi \hbar^{2}}{m} a_{\mathrm{dd}}$ is the dipolar coupling constant, $\mu$ is the magnetic dipole moment, $\theta$ is the angle between $\mathbf{r}$ and the vertical axis (polarization axis of the dipoles), and $a_{\mathrm{dd}}=\frac{\mu_{0} \mu^{2} m}{12 \pi \hbar^{2}}$ is the dipolar length. The parameter $\varepsilon_{\mathrm{dd}}=a_{\mathrm{dd}} / a_{s}$ is the ratio of dipolar interaction to the $s$ wave interaction strengths, defining stability of a uniform condensate in the Bogoliubov approach when $\varepsilon_{\mathrm{dd}}<1$ [5]. Quantum fluctuation corrections to the mean-field energy for a uniform dipolar condensate are introduced in Eq.(1) by a Lee-Huang-Yang-type (LHY) term, with coefficient $g_{\mathrm{LHY}}=\frac{128 \sqrt{\pi}}{3} \frac{\hbar^{2} a_{s}^{5 / 2}}{m}\left(1+\frac{3}{2} \varepsilon_{\mathrm{dd}}^{2}\right)$ [27, [28, 49]. Energetic stability of dipolar droplets in trapping potentials have been studied in a number of works. A stability diagram for droplets in free space was proposed in [26] via a Gaussian ansatz and checked against numerical simulations. The variational approach well describes collective properties of the condensate, such as the energy, the shape close to the instability region, and excitation spectra 29.

\section{ENERGETIC STABILITY DIAGRAM}

We begin our study with the static properties of vortex lines introducing a variational wave function

$$
\psi_{V}(\mathbf{r})=\left(\frac{2^{2 \ell+3} N}{\pi^{\frac{3}{2}} \sigma_{\rho}^{2 \ell+2} \sigma_{z}}\right)^{\frac{1}{2}} \rho^{\ell} e^{i \ell \phi} e^{-2\left(\frac{\rho^{2}}{\sigma_{\rho}^{2}}+\frac{z^{2}}{\sigma_{z}^{2}}\right)},
$$

where $N$ is the particle number and $\rho$ the radial coordinate [50]. The choice $\ell=0$ corresponds to a state with no vortex, whereas for $\ell>0$ the state has $\ell$ quanta of circulation. In this work we specialize to the case of $\ell=1$, and leave the investigation of multicharged vortices to a separate study. In Eq. (2) the widths of the condensate $\sigma_{\rho}$ and $\sigma_{z}$ are variational parameters to be determined via a minimization of the full energy functional associated with Eq. (1). Therefore, we compute the rescaled energy

$$
\begin{aligned}
\frac{E_{V}}{E_{0}}= & \frac{1}{N \sigma_{z}^{2}}\left(1+\frac{4}{y^{2}}\right)+\frac{2 \sqrt{2}}{\pi^{\frac{1}{2}} \sigma_{z}^{3} y^{2} N}\left(\varepsilon_{\mathrm{dd}}^{-1}-g(y)\right) \\
& +\frac{8192 \sqrt{2}}{625 \pi^{5 / 4}} \frac{1+\frac{3}{2} \varepsilon_{\mathrm{dd}}^{2}}{\varepsilon_{\mathrm{dd}}^{5 / 2} N^{2}}
\end{aligned}
$$

where $E_{0}=\frac{\hbar^{2}}{m a_{\mathrm{dd}}^{2}}, y=\sigma_{\rho} / \sigma_{z}, g(x)=f(x)+3 x f^{\prime}(x) / 8+$ $x^{2} f^{\prime \prime}(x) / 8$, and $f(x)=\frac{1+2 x^{2}}{1-x^{2}}-\frac{3 x^{2} \operatorname{arctanh} \sqrt{1-x^{2}}}{\left(1-x^{2}\right)^{3 / 2}}$ [5]. The resulting minimization of Eq. (3) is shown in Fig. 1(a) as a function of $\varepsilon_{\mathrm{dd}}^{-1}$ and particle number $N$. For comparison we show the result of energy minimization for $\ell=0$ (dashed line) [26. The shaded region below the full line is the energetic stability region of a droplet with $\ell=1$, which is shrunk compared to the $\ell=0$ case. Above the solid line there is no minimum, and the minimum energy state is a uniform solution with vanishing density [51].

\section{SHAPE OF DIPOLAR DROPLETS WITH A VORTEX LINE}

We proceed by characterizing the shape of the droplet in the presence of a vortex for different particle numbers and $\varepsilon_{\mathrm{dd}}$. In Fig. 11(b) we compute the vortex core 
(a) $t=0 t_{0}$

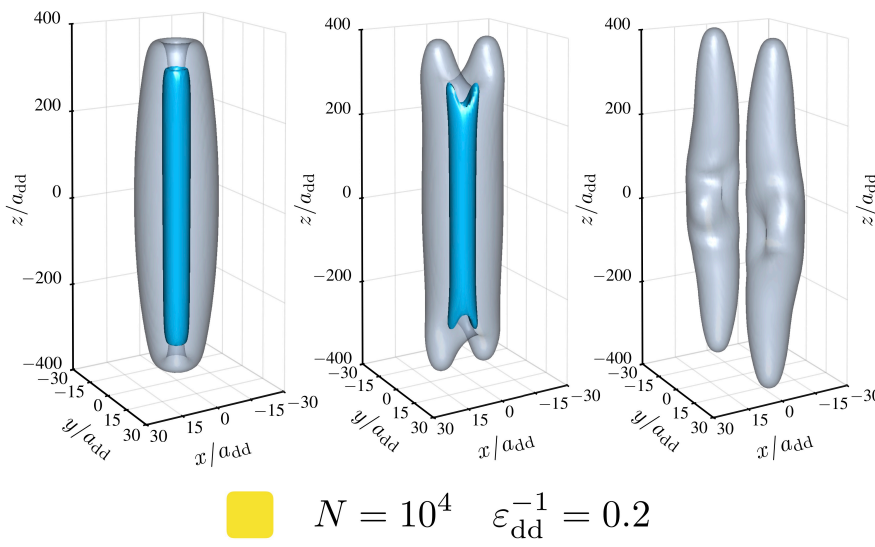

(d) $t=0 t_{0}$

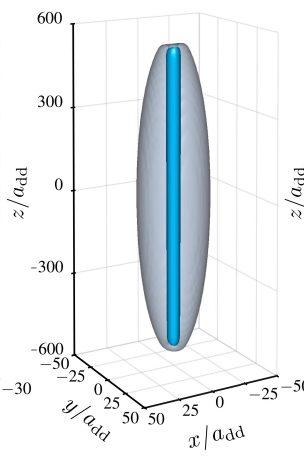

(e) $t=600 t_{0}$

(f) $t=12000 t_{0}$

FIG. 2. Real-time dynamics of droplets with vortex lines. (a), (b), (c) Splitting instability for $N=10^{4}$ particles and $\varepsilon_{\mathrm{dd}}^{-1}=0.2$. Snapshots of the dynamics at $t / t_{0}=0,1200,2000$, where $t_{0}=m a_{\mathrm{dd}}^{2} / \hbar$. At intermediate time $t=1200 t_{0}$ droplets split in two fragments moving at a finite (opposite) momentum and carry excitations with finite angular momentum. (d), (e), (f) Vortex-line bending for $N=10^{5}$ and $\varepsilon_{\mathrm{dd}}^{-1}=0.1$. Snapshots of the dynamics at $t / t_{0}=0,600,12000$. At initial times unstable Kelvin-wave modes tend to split the droplet. After a transient dynamics, where part of the angular momentum is transferred to surface collective excitations, the vortex line bends $\left(t=12000 t_{0}\right)$. See the full-dynamics video in the Supplemental Material [68. Notice the difference in the length scales between the axes of the $N=10^{4}$ and $N=10^{5}$ plots due to very strong anisotropy and different droplet lengths. Results are obtained by solving numerically Eq. (1) on a grid size of $512 \times 512 \times 256$ points.

size in units of $a_{\mathrm{dd}}$, whereas in Fig. 1(c) we plot the horizontal and vertical widths $w_{r}$ and $w_{z}$. The vortex core size is defined from the origin over the $z=0$ plane to the point where the density reaches $90 \%$ of its maximum value. The droplet radial (axial) width is defined as the mean length $w_{r(z)}=\left(w_{i}+w_{e}\right) / 2$, where $w_{i}$ and $w_{e}$ are the distances between the points where the density reaches, respectively, $90 \%$ and $10 \%$ of its maximum value along the radial direction (along the vertical, peakdensity line). Variational calculations are checked against full numerical simulations of Eq. (1) in imaginary time (dots in Fig. 1), exploiting the azimuthal symmetry of vortex states, where we assume $\psi_{\mathrm{V}}(\mathbf{r})=e^{i \phi} \Psi_{\mathrm{V}}(\rho, z)$. The problem reduces then to an effective two-dimensional (2D) problem for the function $\Psi(\rho, z)$ (solved over a grid size of $512 \times 256$ points). To efficiently compute the kinetic term, we employ a discrete Hankel transform in the radial direction (of first order, to impose the vortex node at $\rho=0$ ) and the usual fast Fourier transform in the longitudinal direction [52. In Figs. 1(b) and 1 (c) lines terminate at the spinodal point of Fig. 1(a) for the corresponding value of $N$. We notice that for a wide range of interactions and particle numbers the vortex core is of the same order of the radial width $w_{r}$, and both are always much smaller than the longitudinal width $w_{z}$. All lengths decrease slightly by increasing $\varepsilon_{\mathrm{dd}}$ for a fixed $N$. For every simulation analyzed in Fig. 1, both $\rho$ and $z$ domains were chosen to be at least twice as large as the maximum extension of the droplet predicted by the variational approach (i.e., $\gtrsim 2 \sigma_{z}$ ). The application of an interaction cutoff (as proposed in 53]) turned out to be irrelevant in these large-domain cases, meaning that the Fourier copies (of the periodically bound numerical box) were sufficiently spaced, making artificial effects of the long-range interaction negligible. We observe agreement between the variational and numerical results for small $\varepsilon_{\mathrm{dd}}$ and particle numbers. A discrepancy for large particle numbers and stronger interactions [as is the case of $\varepsilon_{\text {dd }}^{-1}<0.2$ and $N=10^{5}$, see Fig. 1(b)] is expected due to the combined effect of the limited validity of the variational ansatz as well as for resolution issues related to the strong anisotropy of the droplet.

\section{REAL-TIME DYNAMICS}

A crucial issue, relevant for the experiments, is the stability of such vortex states in self-bound droplets. To address this point we perform fully three-dimensional simulations in real time to take into account possible instabilities which can break azimuthal symmetry (see below). The input states at $t=0$ are created from the results of $2 \mathrm{D}$ imaginary-time relaxation described previously. These $2 \mathrm{D}$ solutions are interpolated to a grid of $512 \times 512 \times 256$ points, thus generating three-dimensional initial states [see Figs. 2(a) and 2(d)], slightly perturbed with numerical noise.

In Fig. 2 we illustrate the prototypical real-time dynamics of a droplet with a vortex line at $t=0$ for two cases of condensates of $N=10^{4}$ and $N=10^{5}$ particles with $\varepsilon_{\mathrm{dd}}^{-1}=0.2$ and 0.1 , respectively. Time is measured in units of $t_{0}=m a_{\mathrm{dd}}^{2} / \hbar$, which equals $0.12 \mu \mathrm{s}$ for ${ }^{164} \mathrm{Dy}$ and $0.03 \mu \mathrm{s}$ for ${ }^{168} \mathrm{Er}$. The blue shaded region is an isosurface cut of the magnitude of the pseudovorticity vector $\boldsymbol{\omega}=\boldsymbol{\nabla} \operatorname{Re}(\psi) \times \boldsymbol{\nabla} \operatorname{Im}(\psi)$. This quantity is tangent to the vortex line along its length and can be used to track 

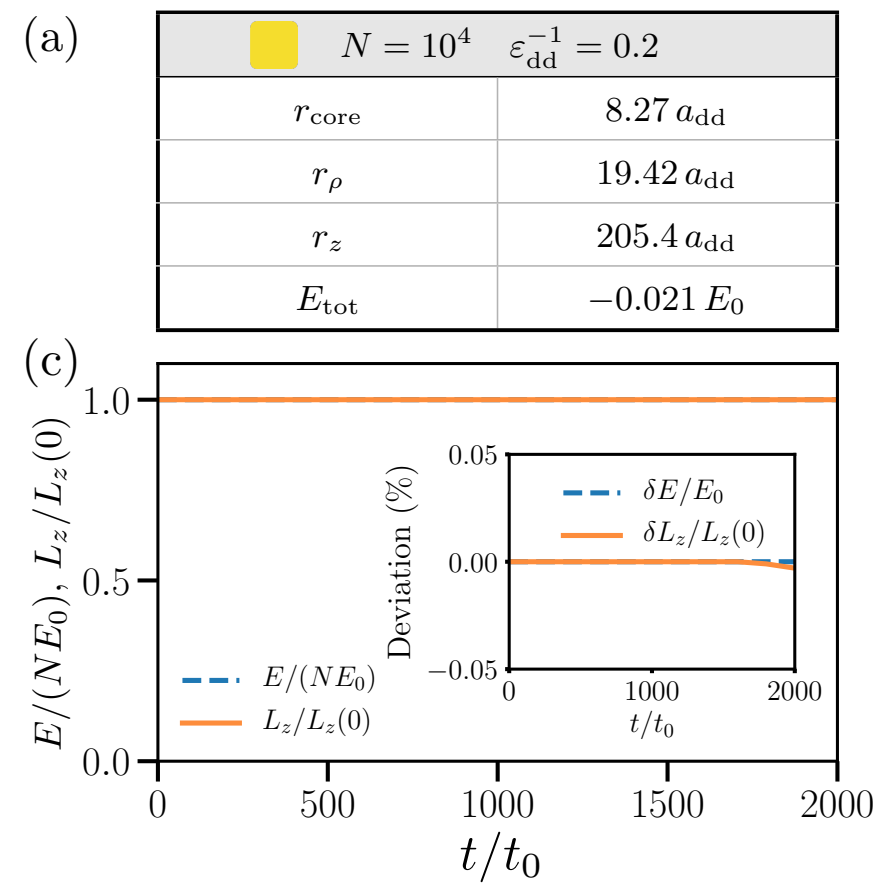

(b)

\begin{tabular}{|c|c|}
\hline$N=10^{5}$ & $\varepsilon_{\mathrm{dd}}^{-1}=0.1$ \\
\hline$r_{\text {core }}$ & $5.82 a_{\mathrm{dd}}$ \\
\hline$r_{\rho}$ & $22.02 a_{\mathrm{dd}}$ \\
$r_{z}$ & $563.1 a_{\mathrm{dd}}$ \\
\hline$E_{\text {tot }}$ & $-0.104 E_{0}$ \\
\hline
\end{tabular}

(d)

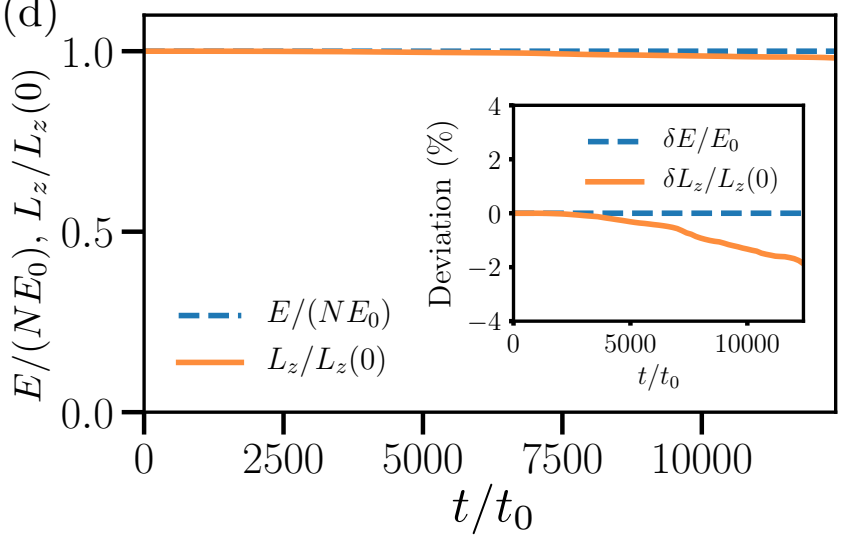

FIG. 3. Numerical simulations. Tables (a) and (b) show the vortex core $r_{\text {core, }}$ the longitudinal $r_{z}$, and the transverse radius $r_{\rho}$ in units of $a_{\mathrm{dd}}$ and the total energy per particle in units of $E_{0}$ for the two points in the diagram of Fig. 1 . They correspond to the stationary state for (a) $N=10^{4}$ and $\varepsilon_{\mathrm{dd}}^{-1}=0.2$, and (b) $N=10^{5}$ and $\varepsilon_{\mathrm{dd}}^{-1}=0.1$. Results are obtained by solving numerically Eq. (1) with imaginary-time evolution on a grid as large as $512 \times 256$ points along the transverse and longitudinal directions, respectively. The domain ranges are (a) $\rho \times z \in[-300,300] \times[-800,800]$ and (b) $\rho \times z \in[-400,400] \times[-1000,1000]$ in units of $a_{\mathrm{dd}}$. Plots (c) and (d) show the energy and longitudinal angular momentum conservation along the dynamics of Fig. 2 for the same particle numbers and interactions as in (a) and (b), respectively.

the numerical points corresponding to the vortex core, where the zeros of the imaginary and real parts of the wave function intersect [54. The two dynamics display very different features. For $N=10^{4}$ the system develops a splitting instability and divides in two droplets with $N_{\mathrm{f}} \approx N / 2$ at $t \approx 1200 t_{0}$, where $N_{\mathrm{f}}$ is the particle number of each droplet after splitting. For longer times the two fragments move apart with opposite momenta and display no residual vorticity. All initial angular momentum gets transferred into collective surface excitations of the droplets. For the larger system, splitting instability starts to develop at the same time $t=600 t_{0}$; however, the droplet does not fragment. At later times the condensate restores a droplet-like configuration with the development of Kelvin waves along the vortex line, eventually leading to vortex bending for $t=12000 t_{0}$ and surface excitations. We observe similar features, i.e., absence of splitting and enhancement of surface excitations, also for longer times. The shape of the bent vortex line resembles the $U$-shaped excitations studied in [55], which were shown to hold less angular momentum than a straightline vortex state, corroborating our description of angular momentum being transferred to surface modes [see Fig. 2 (f)]. Since external torques on the droplet are absent, no change of the angular momentum along any direction is expected. Its necessary conservation means that angular momentum had to be transferred from the vortex line to surface modes. Numerically, a finer grid and larger simulation box would be needed in order to preserve the angular momentum along the vertical direction for very long times. In Figs. 3 (c) and 3(d) we show the numerical deviation of the energy and longitudinal angular momentum for the two cases discussed above.

\section{EXPERIMENTAL CONSIDERATIONS}

Vortices in ultracold atomic systems are controllably created either by phase imprinting or via an effective rotating potential generated by an applied laser beam. In any case, vortex imprint must be done in-trap, following the droplet preparation and before its release. The optical spoon technique is more invasive and requires longer equilibration times and it becomes less likely to work experimentally. Phase imprinting, on its turn, can be done with high spatial resolution as well as by means of very short optical pulses, allowing an almost instantaneous imprint of a vortex. Following the simulations presented above, the typical times for the observation of vortex dynamics for ${ }^{164}$ Dy range from $100 \mu$ s to just below $2 \mathrm{~ms}$, which is short but still within experimental resolution. For example, for the parameters as in Fig. 2(a), splitting instability sets on for times $t \approx 10^{3} t_{0}$, which correspond to $t \approx 0.1 \mathrm{~ms}$ for ${ }^{164} \mathrm{Dy}$. Nevertheless, we have also veri- 
fied instances in which the onset of the splitting process took slightly longer time. This was the case of $\varepsilon_{\mathrm{dd}}^{-1}=0.6$ and $N=10^{5}$, where $t \approx 45000 t_{0}$, corresponding to $t \approx 6 \mathrm{~ms}$. Detection, in any case, must be done in situ, preferably with a levitating external magnetic field gradient to allow for longer observation times [21]. Extraction of dynamical properties, e.g., momentum, shape, atomnumber, and/or absence of droplet movement in the cases covered in Fig. 2, can be done at longer evolution times, on the order of several milliseconds, when detection is expected to be easier.

\section{CONCLUSIONS}

In this article we studied the stability of quantum vortex lines in dilute self-bound droplets of dipolar atoms. We first discussed the energetic stability region of such vortex excitations via a variational ansatz in the generalized nonlocal Gross-Pitaevskii functional that includes a LHY-type contribution. The region corresponding to the stationary solutions where $E_{\mathrm{V}}<0$ is unstable to fragmentation into two droplets. When this is not the case we found that Kelvin waves establish along the vortex line, which eventually bends in the central region of the droplet. We confirmed our findings by detailed fully three-dimensional numerical simulations of vortex states created by phase imprinting. The situation where Kelvin waves start developing has also been predicted to appear in a similar context of three-dimensional dipolar BECs [11. Droplets with vortices may thus serve as promising test-beds to the study of twisted vortex lines in real-life experiments. An extension of this work would include the investigation of the excitation spectrum of these vortex lines, similarly to what has been recently done in vortex-free droplets [30, and with vortex states in trapped geometries [56]. These instabilities offer new opportunities for devising stabilization methods, such as temporal or spatial modulation of the scattering length as proposed for nondipolar BECs [57, 58 or pinning potentials [59, 60]. Also, the appearance of vortex arrays 61, 62 as well as the effects of impurities and turbulence phenomena may be relevant to current experiments 63 66.

\section{ACKNOWLEDGMENTS}

We thank V. S. Bagnato for valuable discussions and A. Cappellaro for carefully reading the manuscript. Support from CePOF through FAPESP 2013/07276-1 is acknowledged. T.M. acknowledges CNPq for support through Bolsa de Produtividade em Pesquisa No. 311079/2015-6. E.A.L.H. acknowledges support from FAPESP through Grant No. 2015/20475-9. F.E.A.S. acknowledges CNPq for support through Bolsa de Produtividade em Pesquisa No. 305586/2017-3. A.C. is supported by FAPESP through Grant No. 2017/09390-7. This research was developed with the help of XMDS2 software 67, making use of the computational resources of the Center for Mathematical Sciences Applied to Industry (CeMEAI) financed by FAPESP.
[1] A. Fetter and A. Svidzinsky, J. Phys.: Condens. Matter 13 R135R194 (2001).

[2] F. Dalfovo and S. Stringari, J. Chem. Phys. 115, 10078 (2001)

[3] K. W. Madison, F. Chevy, W. Wohlleben, and J. Dalibard Phys. Rev. Lett. 84, 806 (2000).

[4] M. W. Zwierlein, J. R. Abo-Shaeer, A. Schirotzek, C. H. Schunck, and W. Ketterle, Nature (London) 435, 10471051 (2005).

[5] T. Lahaye, C. Menotti, L. Santos, M. Lewenstein, and T. Pfau, Rep. Prog. Phys. 72, 126401(2009).

[6] A M Martin, N G Marchant, D H J ODell and N G Parker, J. Phys.: Condens. Matter 29, 103004 (2017).

[7] M. Abad, M. Guilleumas, R. Mayol, M. Pi, and D. M. Jezek, Phys. Rev. A 79, 063622 (2009).

[8] I. Tikhonenkov, B. A. Malomed, and A. Vardi, Phys. Rev. A 78, 043614 (2008).

[9] R. M. Wilson, S. Ronen, and J. L. Bohn, and H. Pu, Phys. Rev. Lett. 100, 245302 (2008).

[10] W. E. Shirley, B. M. Anderson, C. W. Clark, and R. M. Wilson, Phys. Rev. Lett. 113, 165301 (2014).

[11] M. Klawunn, R. Nath, P. Pedri, and L. Santos, Phys. Rev. Lett. 100, 240403 (2008).

[12] M. Klawunn and L. Santos, New Journal of Physics 11, 055012 (2009).
[13] F. Dalfovo, S. Giorgini, L. P. Pitaevskii, and S. Stringari Rev. Mod. Phys. 71, 463 (1999).

[14] N. Navon, S. Piatecki, K. Günter, B. Rem, T. C. Nguyen,1 F. Chevy, W. Krauth, and C. Salomon, Phys. Rev. Lett. 107, 135301 (2011).

[15] N. Navon, S. Nascimbène, F. Chevy, C. Salomon, Science 328, 729 (2010).

[16] H. Kadau, M. Schmitt, M. Wenzel, C. Wink, T. Maier, I. Ferrier-Barbut, and T. Pfau, Nature (London) 530, 194 (2016).

[17] I. Ferrier-Barbut, H. Kadau, M. Schmitt, M. Wenzel, and T. Pfau, Phys. Rev. Lett. 116, 215301 (2016).

[18] L. Chomaz, S. Baier, D. Petter, M. J. Mark, F. Wächtler, L. Santos, and F. Ferlaino, Phys. Rev. X 6, 041039 (2016).

[19] I. Ferrier-Barbut, M. Schmitt, M. Wenzel, H. Kadau, and T. Pfau, J. Phys. B 49, 214004 (2016).

[20] M. Schmitt, M. Wenzel, B. Boöttcher, I. Ferrier-Barbut, and T. Pfau, Nature 539, 259 (2016).

[21] M.Wenzel,F.Böttcher,T.Langen,I.Ferrier-Barbut, and T. Pfau, Phys. Rev. A 96, 053630 (2017).

[22] L. Chomaz et al., Nature Physics 14, 442-446 (2018)..

[23] C. R. Cabrera, L. Tanzi, J. Sanz, B. Naylor, P. Thomas, P. Cheiney, and L. Tarruell, Science 359, 301 (2018).

[24] D. S. Petrov, Phys. Rev. Lett. 115, 155302 (2015). 
[25] D.S. Petrov and G. E. Astrakharchik, Phys. Rev. Lett. 117, 100401 (2016).

[26] D. Baillie, R. M. Wilson, R. N. Bisset, and P. B. Blakie, Phys. Rev. A 94, 021602(R) (2016).

[27] F. Wächtler and L. Santos, Phys. Rev. A 93, 061603 (2016).

[28] R. N. Bisset, R. M. Wilson, D. Baillie and P.B. Blakie, Phys. Rev. A 94, 033619 (2016).

[29] F. Wächtler and L. Santos, Phys. Rev. A 94, 043618 (2016).

[30] D. Baillie, R. M. Wilson, and P. B. Blakie, Phys. Rev. Lett. 119, 25302 (2017).

[31] F. Cinti and M. Boninsegni, Phys. Rev. A 96, 013627 (2017).

[32] A. Macia, J. Sánchez-Baena, J. Boronat and F. Mazzanti, Phys. Rev. Lett. 117, 205301 (2016).

[33] F. Cinti, A. Cappellaro, L. Salasnich, T. Macrì, Phys. Rev. Lett. 119, 215302 (2017).

[34] H. Saito, Journal of the Physical Society of Japan 85, 053001 (2016).

[35] A. Boudjemâa, Annals of Physics 3816879 (2017).

[36] D. Laghi, T. Macrì, A. Trombettoni, Phys. Rev. A. 96, 043605 (2017).

[37] A. Cappellaro, T. Macrì, G. F. Bertacco, L. Salasnich, Scientific Reports, 7, 13358 (2017).

[38] For similar results that include the stabilization via non dissipative three-body interactions see, e.g., S. K. Adhikari, Laser Phys. Lett. 14, 025501 (2017); Phys. Rev. A 95, 023606 (2017).

[39] L. F. Gomez, K. R. Ferguson, J. P. Cryan, C. Bacellar, R. M. P. Tanyag, C. Jones, S. Schorb, D. Anielski, A. Belkacem, C. Bernando et al., Science 345, 906 (2014).

[40] F. Dalfovo, R. Mayol, M. Pi, and M. Barranco, Phys. Rev. Lett. 85, 1028 (2000).

[41] K. K. Lehmann and Roman Schmied, Phys. Rev. B 68, 224520 (2003).

[42] F. Ancilotto, M. Pi, and M. Barranco., Phys. Rev. B 91, 100503 (2015).

[43] F. Ancilotto, M. Barranco, F. Coppens, J. Eloranta, N. Halberstadt, A. Hernando, D. Mateo and M. Pi, International Reviews in Physical Chemistry, 36:4, 621-707 (2017).

[44] H. Saito and M. Ueda, Phys. Rev. Lett. 89, 190402 (2002).

[45] A. Aftalion and T. Riviere, Phys. Rev. A 64, 043611 (2001).

[46] J. J. García-Ripoll and V. M. Pérez-García, Phys. Rev. A 64, 053611 (2001).

[47] V. Bretin, P. Rosenbusch, F. Chevy, G.V. Shlyapnikov, and J. Dalibard, Phys. Rev. Lett. 90, 100403 (2003).
[48] T. P. Simula, T. Mizushima, and K. Machida, Phys. Rev. Lett. 101, 020402 (2008).

[49] A. R. P. Lima and A. Pelster, Phys. Rev. A 84, 041604(R) (2011).

[50] R. P. Teles, F. E. A. dos Santos, M. A. Caracanhas, and V. S. Bagnato, Phys. Rev. A 87, 033622 (2013).

[51] We checked from the variational ansatz that metastable solutions for which a local minimum has positive energy exist and are within the width of the black solid line.

[52] The Hankel transform of a function $f(\rho, z) e^{i \ell \phi}$ is given by $\tilde{f}\left(k_{\rho}, k_{\phi}, z\right)=-2 i \pi \int_{0}^{\infty} f(\rho, z) J_{1}\left(k_{\rho} \rho\right) \rho d \rho$. The full momentum-space representation of $f(\rho, z) e^{i \ell \phi}$ is then $\tilde{f}(\mathbf{k})=\mathcal{F}_{z}\left[\tilde{f}\left(k_{\rho}, k_{\phi}, z\right)\right]$, with $\mathcal{F}_{z}[\cdot]$ the usual Fourier transform along the $z$-direction. See [56] for further details on the algorithm.

[53] H. Y. Lu, H. Lu, J. N. Zhang, R. Z. Qiu, H. Pu, and S. Yi, Phys. Rev. A 82, 023622 (2010).

[54] S. Serafini, L. Galantucci, E. Iseni, T. Bienaimé, R. N. Bisset, C. F. Barenghi, F. Dalfovo, G. Lamporesi, and G. Ferrari, Phys. Rev. X 7, 021031 (2017).

[55] A. Aftalion, and I. Danaila Phys. Rev. A 68, 023603 (2003).

[56] R. M. Wilson, S. Ronen, and J. L. Bohn, Phys. Rev. A 79, 013621 (2009).

[57] F. K. Abdullaev, J. G. Caputo, R. A. Kraenkel, and B. A. Malomed, Phys. Rev. A, 67, 013605 (2003).

[58] S. K. Adhikari, Phys. Rev. A 69, 063613 (2004).

[59] T. Isoshima and K. Machida, Phys. Rev. A 59, 2203 (1999).

[60] P. Kuopanportti, J. Low. Temp. Phys. 161, 56, pp. 561573 (2010).

[61] N. R. Cooper, E. H. Rezayi, and S. H. Simon, Phys. Rev. Lett. 95, 200402 (2005).

[62] J. Zhang and H. Zhai, Phys. Rev. Lett. 95, 200403 (2005).

[63] C. F. Barenghi, L. Skrbek, and K. R. Sreenivasan, PNAS 111, 4647 (2014)

[64] M. C. Tsatsos, P. E. S. Tavares, A. Cidrim, A. R. Fritsch, M. A. Caracanhas, F. E. A. dos Santos, C. F. Barenghi, and V. S. Bagnato, Phys. Rep. 622 1-52 (2016).

[65] M. Tsubota, K. Fujimoto, S. Yui, J. Low. Temp. Phys. 188, 119 (2017).

[66] T. Bland, A. W. Baggaley, N. G. Parker, arXiv: 1706.02660

[67] G. R. Dennis, J. J. Hope, and M. T. Johnsson, Comput. Phys. Commun. 184, 201-208 (2013).

[68] For the full dynamics see the Supplemental Material at URL to be provided by publisher. 